\title{
PERFORMANCE ANALYSIS OF A DYNAMIC CHANNEL ALLOCATION TECHNIQUE FOR SATELLITE MOBILE CELLULAR NETWORKS
}

\author{
ENRICO DEL RE, ROMANO FANTACCI AND GIOVANNI GIAMBENE
Dipartimento di Ingegneria Elettronica, Università di Firenze, Via S. Marta, 350139 Firenze, Italy
}

\begin{abstract}
SUMMARY
This paper deals with an efficient dynamic channel allocation (DCA) technique suitable for applications in mobile satellite cellular networks. A cost function is defined to allow an optimum selection of channels to be allocated on demand. A mobility model suitable for low earth orbit (LEO) satellite has been derived by The performance of the novel DCA technique in terms of call blocking probability has been derived by simulations. The obtained results are compared with those achieved by a fixed channel allocation (FCA) technique to show a better behaviour.
\end{abstract}

KEY WORDS Personal communications Mobile networks Dynamic channel allocation

\section{INTRODUCTION}

The rapid growth in the demand for personal communications has led to the need for intense research and development efforts towards a new generation of Personal Communication Systems (PCSs). Any future PCS will integrate different services to provide data and voice communications via a multimedia portable terminal. The challenge is to build a personal communication network (PCN) extended on a world-wide basis (Universal PCN) which allows the transfer of information (data, voice, etc.) in a reliable way between two users, at any points on the earth, and either in fixed positions or travelling by train or by aircraft at hundreds of kilometres per hour

In this scenario, mobile satellite systems (MSSs) which permit a global coverage of the earth to be achieved (also including impervious and scarcelypopulated areas) and internetworking with the existing terrestrial mobile networks, will play a very important role.

This paper is focused on a global coverage MSS, using satellites with on-board signal processing capabilities, which is mainly devoted to phone communications.

One of the major issues for implementing an efficient MSS is to allow the large demand consistent with limited bandwidth and high-quality service. In particular, the primary concern is the selection of the satellite's orbit. This paper deals with the case of geostationary (GEO) satellites and the case of low earth orbit (LEO) satellites.

Theoretically, we need three GEO satellites to cover all the earth; however, since the satellite visual angle from a mobile user reduces when increasing the latitude, the link is reliable for North and South latitudes within $\pm 70^{\circ}$. Another difficulty is the remarkable distance of the GEO satellite from the earth* which demands high transmission power from the earth terminals, making the realization of small sized terminals extremely difficult.

To overcome these drawbacks it is possible to employ satellites with orbits (Figure 1) within $500 \mathrm{~km}$ and $2000 \mathrm{~km}$ (LEOs). These satellites have a motion relative to the earth so that several satellites alternatively serve the same terrestrial area. For this reason a constellation formed by many LEO satellites is required in order to obtain a global earth coverage. In particular, the required number of

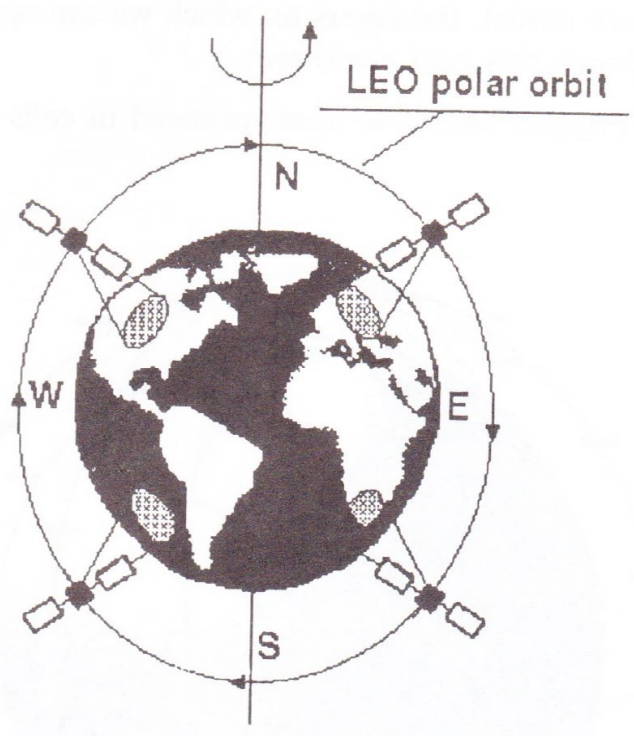

Figure 1. LEO polar satellites

\footnotetext{
* The orbit of a GEO satellite is on the equatorial plane at about $36,000 \mathrm{~km}$ altitude.
} 
LEO satellites decreases when the altitude of their orbit increases.

This paper deals with two particular LEO systems which achieve a good global coverage, also including polar areas: the IRIDIUM system (by Motorola Inc.) and the ARIES system (by Constellations Communications).

The IRIDIUM system (Figure 2) employs 66 LEO satellites over six polar orbits at about $780 \mathrm{~km}$ altitude, whereas the ARIES system employs 48 LEO satellites over four polar orbits at about $1000 \mathrm{~km}$ altitude. ${ }^{1}$

In any MSS the geographical area served is divided into cells. Each cell is illuminated by a radiation spot-beam of a satellite. For LEO satellites, at relatively low altitude, the resulting cells have smaller sizes than the ones obtained by GEO satellites. However, in the first case we obtain a more widespread territory division with remarkable advantages for the traffic carried by the network.

A limiting factor is the co-channel interference due to simultaneous use of the same channel in different spatial cells. Considering a mobile communication system with cellular coverage given by equal-size hexagonal cells, in order to reduce the co-channel interference the same channel cannot be simultaneously used in cells whose centres are distant less than the reuse distance, $D .^{2}$ These cells form the belt of interfering cells.

The derivation of $D$ depends on several factors, including the particular multiple access technique and modulation scheme used, mobile environment and acceptable voice quality. The performance of a mobile communication system is increased by a better use of available channels. Referring to the OSI network model, the layers on which we can operate to achieve this goal are three:

\section{Physical layer: we must proceed in cells split-}

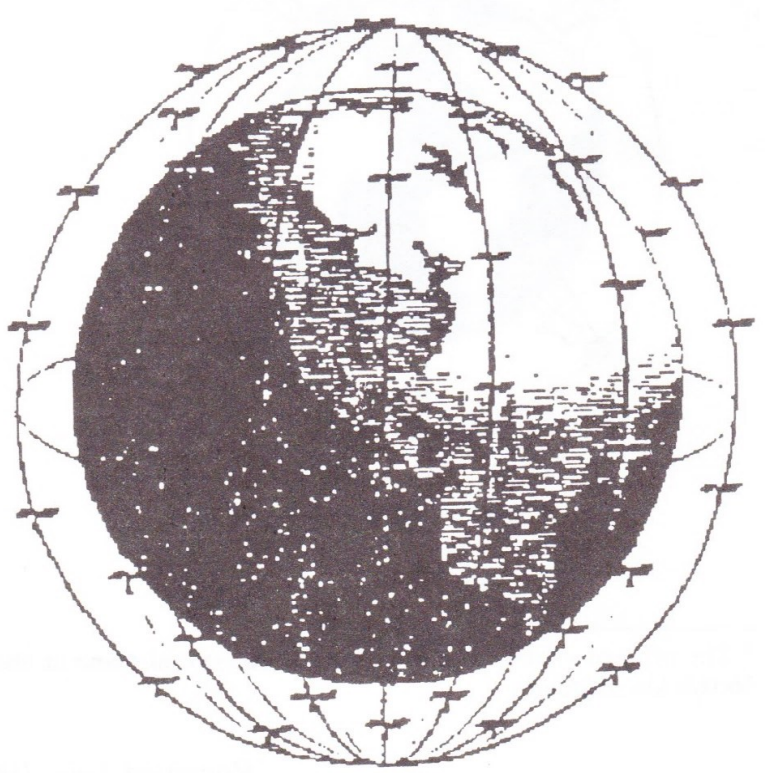

Figure 2. IRIDIUM satellite system ting, keeping unvaried their capacity, so that the quantity of traffic carried on by the network increases $\left(\propto 1 / R^{2}\right.$, with $R$ denoting the hexagonal cells side). In a MSS, this means that the satellite's orbit must be carefully selected.

2. Data link layer: we must choose the multiaccess technique which is able to grant the available spectrum to the utmost possible number of simultaneous users.

3. Network layer: a suitable dynamic channel allocation (DCA) technique can be used to allow a better use of network resources. ${ }^{3}$

In this paper we shall focus on the network layer. Differently from previously proposed MSSs (e.g. the IRIDIUM system), where channels are assigned to each cell in a fixed way (FCA), an efficient technique for the assignment of channels on demand (DCA) is considered.

Using the proposed DCA technique, whenever a new call attempt arrives in a cell (i.e. satellite antenna spot-beam footprint), the territorial competent satellite executes the dynamic channel allocation algorithm. The channel to be assigned is selected on the basis of a suitable cost function depending on the reuse distance and on the number of interfering cells in which each available channel is just locked. The satellite decides, on a call-bycall basis, the channel to be allocated by exhaustive search for the minimum cost channel. In addition to this, an efficient channel rearrangement technique has also been considered whenever a call termination or handover occurs in a cell. It has also been assumed that satellites can exchange control information about the availability of channels in the cells on the boundaries of the regions they manage. In the case of LEO satellite constellations, this involves the use of inter-satellite links (ISLs), so that a satellite can communicate with the neighbouring ones.

The performance of the DCA technique under consideration has been derived by considering that

(a) the maximum number of available channel is equal to $M$

(b) call duration is random and exponentially distributed, with expected value equal to $T_{\mathrm{m}}$

(c) blocked calls are cleared

(d) the offered traffic per cell is Poisson distributed with the same mean arrival rate $\lambda$ in all the cells.

A handover procedure takes place every time a mobile subscriber (MS) with a call in progress passes from a cell to an adjacent one. In this new cell in order to avoid a forced call termination a new channel must be assigned.

In the GEO case the satellites are stationary with respect to the earth and the change of the cell for a MS is only due to the user's motion. Since the cells have wide sizes, the handover probability is 
very low. Starting from this consideration, we have assumed for the GEO system fixed users only.

In the LEO case (Figure 3), considering the high value of the satellite's oribital speed $\left(V_{\text {orb }}\right)$, we can neglect both the MS speed and the earth rotation speed ( $V_{\text {earth }} ; V_{\text {earth }} \approx 1670 \mathrm{~km} / \mathrm{h}$ ), therefore resulting in an MS's constant speed (relative to the satellites) equal to $V_{\text {orb }}$ (about $22,500 \mathrm{~km} / \mathrm{h}$ ).

Taking into account the high value of the MS's (relative) speed, it is evident that in the LEO case, a call in progress gives rise to several handovers. For this reason the use of the proposed DCA technique becomes of fundamental importance to guarantee a low probability that a call in progress be dropped.

The organization of this paper is as follows: Section 2 describes the proposed DCA technique; in Section 3 handover and user mobility are discussed in the LEO case; finally, in Section 4 simulation results concerning the performance of the proposed DCA technique in terms of call blocking probability are presented and compared with those obtained by means of an FCA technique either in the GEO case, with fixed users, or in the LEO case, with mobile users.

\section{DYNAMIC CHANNEL ALLOCATION TECHNIQUE}

In this section an efficient (DCA) technique is presented. The proposed DCA technique achieves an efficient spectrum utilization by allowing the use of each of the $M$ channels in any cell on the condition that the constraint on $D$ is respected.

We remark that in a mobile communication system $D$ represents the minimum distance between cells for which the use of the same channel gives rise to an acceptable co-channel interference level.

Let us consider a particular cell $x$ and denote by $I(x)$ the set of cells interfering with $x$, i.e. those cells for which it is not permitted to allocate any channel just used in $x$. Note also that the vice versa is valid. Figure 4 shows the cells in $I(x)$ for the

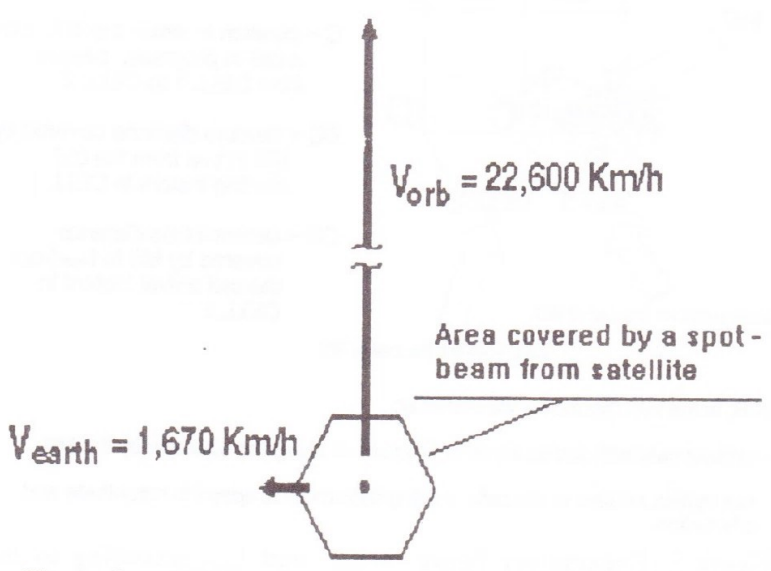

Figure 3. LEO satellite system: main speed components case $D=\sqrt{ } 21 R$, with $R$ the hexagonal cell's side. ${ }^{4}$ From above, it is evident that a channel $i$ is available for allocation to the cell $x$ if it is not just used either in $x$ or in $I(x)$

Let us denote by $\Lambda(x)$ the set of available channels for the cell $x$ at the call arrival instant in $x$. For each channel $i \in \Lambda(x)$, we define the following cost function:

$$
C_{x}(i) \triangleq \sum_{k \in I(x)}\left\{u_{k}(i)\right\}
$$

where

$$
u_{k}(i)= \begin{cases}1, & \text { if } i \in \Lambda(k) \\ 0, & \text { otherwise }\end{cases}
$$

In other words, the cost function $C_{x}(i)$ represents the number of interfering cells of $x$ that can use the channel $i$. Whenever a new call arrives in $x$, if $\Lambda(x) \neq \varnothing$, the channel $i^{*}$ which satisfies the following condition:

$$
i^{*}: C_{x}\left(i^{*}\right)=\min _{i \in \Lambda(x)}\left\{C_{x}(i)\right\}
$$

is temporarily assigned to $x$. Note that the selection of channel $i^{*}$ according to (3) permits the minimization of the number of cells in $I(x)$ in which the use of the same channel $i^{*}$ will be locked, and therefore an increase in the spectrum utilization.

If more channels satisfy (3) a random choice is performed.

In the case of $\Lambda(x)=\varnothing$ (i.e. no channel can be temporarily assigned to the cell $x$ ), the call attempt is blocked and lost. The updating of $\Lambda(x)$, and therefore the evaluation of the cost $C_{x}(i)$ for any

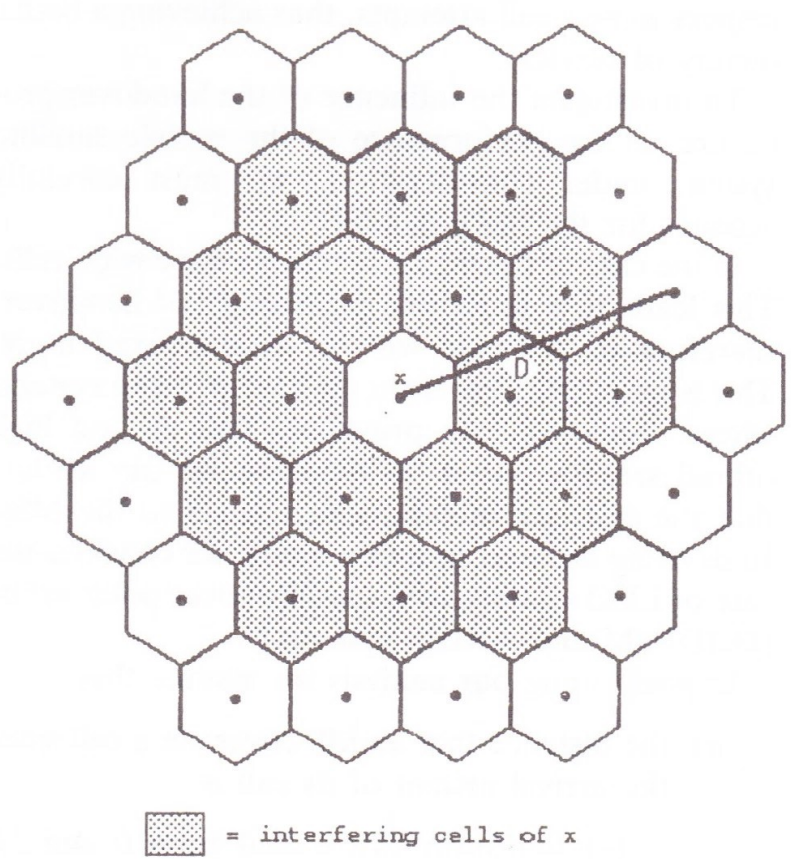

Figure 4. Interfering cells 
channel $i \in \Lambda(x)$, is performed on the basis of tables which take into account the channel's availability in all the cells of the network. Assuming satellites with on-board signal processing capabilities, these tables are handled by the satellite. This permits the attainment of a distributed implementation of the DCA algorithm, in particular in the case of LEO satellite systems.

In order to obtain a better performance, whenever we have a call termination in a cell, the DCA technique executes a channel rearrangement to de-allocate the channel which becomes available in the greatest number of cells in $I(x)$.

\section{HANDOVER AND USER MOBILITY}

In several mobile satellite systems, such as the LEO systems, the handover procedure has a significant impact on the system performance.

Handover can occur in the time interval during which the ratio of the power levels received from the antenna spot-beam serving the current cell and the spot-beam serving the destination cell of the mobile is within two appropriate thresholds. ${ }^{5}$ The handover procedure gives rise to a call termination in the current cell jointly with a new call arrival in the destination cell.

Different handover policies have been proposed recently. Some of them prioritize handovers at the expense of an increased call blocking probability for new arrivals. The prioritization schemes are motivated by the fact that from the MS point of view, forced termination of an ongoing call is much less desirable than blocking a new call attempt.

This paper deals with handover policy which manages handover requests in the same manner as new call attempts. However, it will be shown by computer simulation that the DCA technique implicitly gives a higher priority to the handover service with respect to new call attempts, thus achieving a better quality of service.

To investigate the influence of the handover procedure on the performance of the mobile satellite system under consideration, we must carefully account for the MS's mobility.

In the case of the GEO system we have wide cells. This leads to a negligible probability of handover; therefore in this case we can assume fixed users. This is quite different from the case of LEO systems where handovers are principally due to the high orbital satellite speed. In this case we can assume that the cells are moving with respect to the MSs. In deriving the user mobility model we consider the case of LEO systems with satellites over polar orbits (IRIDIUM and ARIES systems).

In performing our analysis we assume that

(a) the distance that an MS covers in a cell since the arrival instant of its call is

(i) uniformly distributed from 0 and $2 R$ (where $R$ is the cell's side), in the cell where the call has been originated (source cell)

(ii) fixed and equal to $2 R$, in any other cell different from the source cell (transit cell)

(b) every MS travels in a cell with a constant velocity (relative to the satellite) equal to the satellite orbital speed $V_{\text {orb }}$

(c) whenever a handover occurs, the destination cell is the adjacent cell in the direction of the satellite motion.

Moreover, we define the following parameter:

$$
\alpha \triangleq \frac{2 R}{V_{\text {orb }} T_{\mathrm{m}}}
$$

to characterize the user mobility ( $\alpha$ decreases when users mobility increases).

Let us denote by $t_{\mathrm{mc} 1}$ (Figure 5) the permanence time of an MS having a call in progress in the source cell. The parameter $t_{\mathrm{mc} 1}$, is a random variable with a probability density function (p.d.f.) given by

$$
\operatorname{pdf}_{t_{\mathrm{mc} 1}}(t)= \begin{cases}\frac{V_{\mathrm{orb}}}{2 R}, & \text { for } 0 \leq t \leq \alpha T_{\mathrm{m}} \\ 0, & \text { otherwise }\end{cases}
$$

Likewise, we denote by $t_{\mathrm{mc} 2}$ (Figure 5 ) the permanence time of an MS having a call in progress in a transit cell. In this case the parameter $t_{\mathrm{mc} 2}$, is deterministic and can be defined as

$$
t_{\mathrm{mc2}}=\frac{2 R}{V_{\mathrm{orb}}}
$$

Let $t_{\mathrm{d}}$ be the call duration time (exponentially distributed with expected value equal to $\left.T_{\mathrm{m}}\right)$. According to our model a handover procedure occurs whenever

(a) $t_{\mathrm{mc1}}<t_{\mathrm{d}}$, for a new call in the source cell
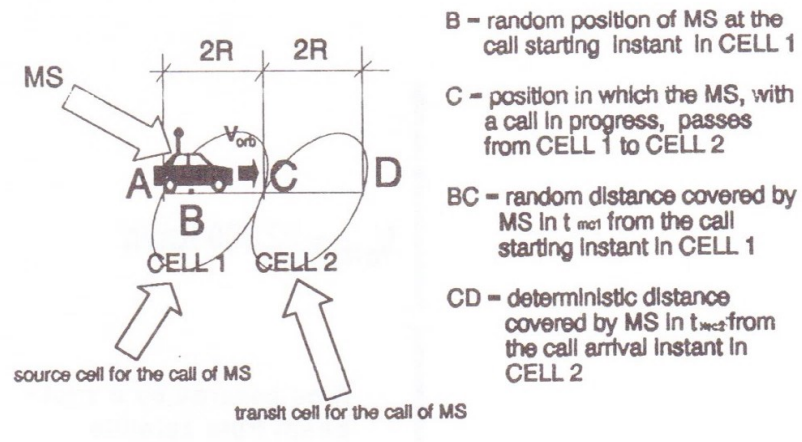

N.B. In the mobility model we consider:

- crrcular cells with radlus equal to $R$, crossed along the dlameter by the MS; - MS motion relative to the cells, having deterministic speed in magnitude and orientation.

Figure 5. Explanatory figure of $t_{\mathrm{mc1}}$ and $t_{\mathrm{mc2}}$ according to the mobility model 
(b) $t_{\mathrm{mc} 2}<t_{\mathrm{d}}$, for a call in the transit cell.

Let us denote by $P_{\mathrm{h} 1}$ the probability that a new call gives rise to a handover and $P_{\mathrm{h} 2}$ the probability that a call in a transit cell gives rise again to a new handover. These probabilites are defined as

$$
\begin{aligned}
P_{\mathrm{h} 1} & =\int_{0}^{\infty} \operatorname{Prob}\left\{t_{\mathrm{d}}>t \mid t_{\mathrm{mc} 1}=t\right\} \operatorname{pdf}_{t_{\mathrm{mc} 1}}(t) \mathrm{d} t \\
& =\frac{1-\mathrm{e}^{-\alpha}}{\alpha} \\
P_{\mathrm{h} 2} & =\operatorname{Prob}\left\{t_{\mathrm{d}}>\frac{2 R}{V_{\text {orb }}}\right\}=\mathrm{e}^{-\alpha}
\end{aligned}
$$

Figure 6 shows $P_{\mathrm{h} 1}$ and $P_{\mathrm{h} 2}$ as a function of $\alpha$. In particular this Figure highlights that as $\alpha$ approaches $0(\infty), P_{\mathrm{h} 1}$ and $P_{\mathrm{h} 2}$ approach $1(0)$.

The probability that a call in progress in a cell may have a handover to an adjacent cell is independent of the number of handovers that the call has already suffered. Under this assumption, the average number of handovers per call attempt, $n_{\mathrm{h}}$, results in

$$
n_{\mathrm{h}}=\frac{\left(1-P_{\mathrm{b} 1}\right) P_{\mathrm{h} 1}}{1-\left(1-P_{\mathrm{b} 2}\right) P_{\mathrm{h} 2}}
$$

where $P_{\mathrm{b} 1}$ is the blocking probability for new call attempts and $P_{\mathrm{b} 2}$ is the dropping probability due to unsuccessful handover.

Figure 7 shows $n_{\mathrm{h}}$ in the case of $P_{\mathrm{b} 1}=P_{\mathrm{b} 2}=0$, as a function of the LEO satellite's altitude, assuming constant the call arrival rate per cell and the half power beam width (HPBW) of the antenna's spot- beams on the satellites. Under this assumption from (9) it follows that the mean number of handovers per call increases as the altitude of the LEO orbits decreases.

\section{RESULTS AND COMPARISONS}

In this section the performance of the DCA technique in terms of call blocking probability is derived and compared with that we obtain by using an FCA technique under the assumption of uniform load conditions for the cells. The cases of LEO and GEO satellite systems are considered.

Figure 8 shows the configuration of the mobile network assumed in evaluating the performance of the DCA technique by computer simulations, with $N$ denoting the number of cells on a side of the network.

This network structure is widely used in the literature $(N=7)^{3,5}$ in deriving the performance of allocation techniques for terrestrial mobile networks. In the DCA case, the cells shown in Figure 8 can be divided into two groups:

(i) edge cells, for which the set of interfering cells is not complete (see Figurc 4)

(ii) central cells, for which the set of interfering cells is complete. These cells are shaded in Figure 8 .

In performing our simulations we have considered only central cells (shaded in Figure 8) having a complete set of interfering cells. Note that a lower call blocking probability usually results if we consider the edge cells (edge effect). ${ }^{6}$

The simulation results presented in this section

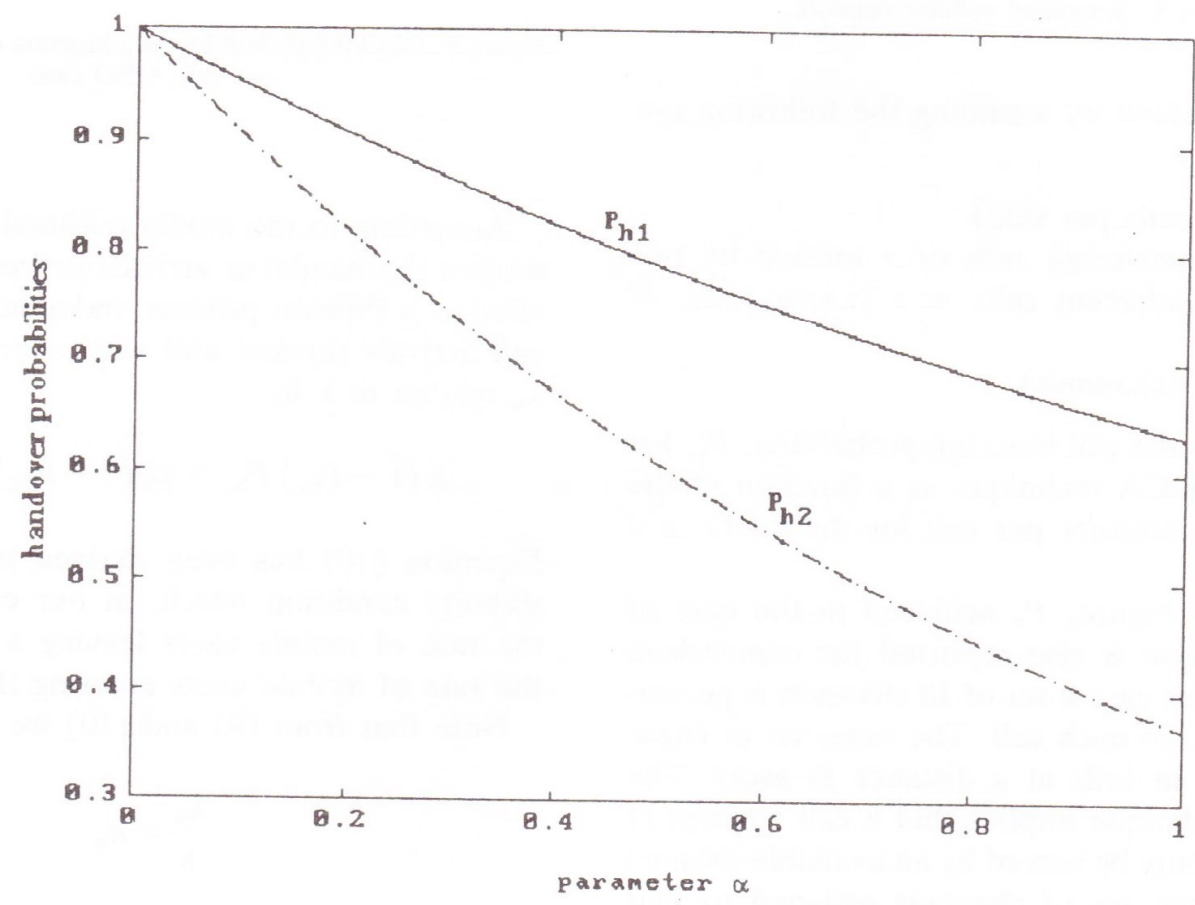

Figure 6. Handover probabilities as a function of $\alpha$ 


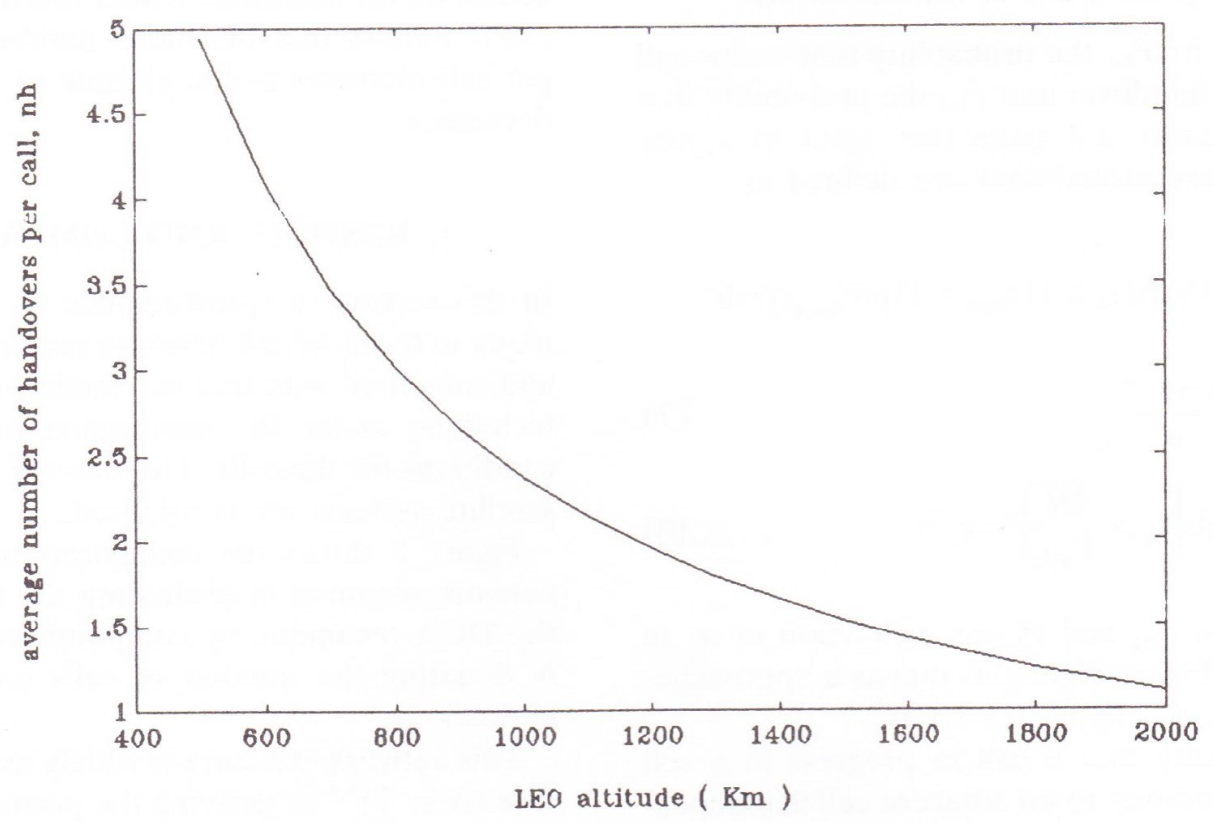

Figure 7. Average number of handovers per call (LEO system)

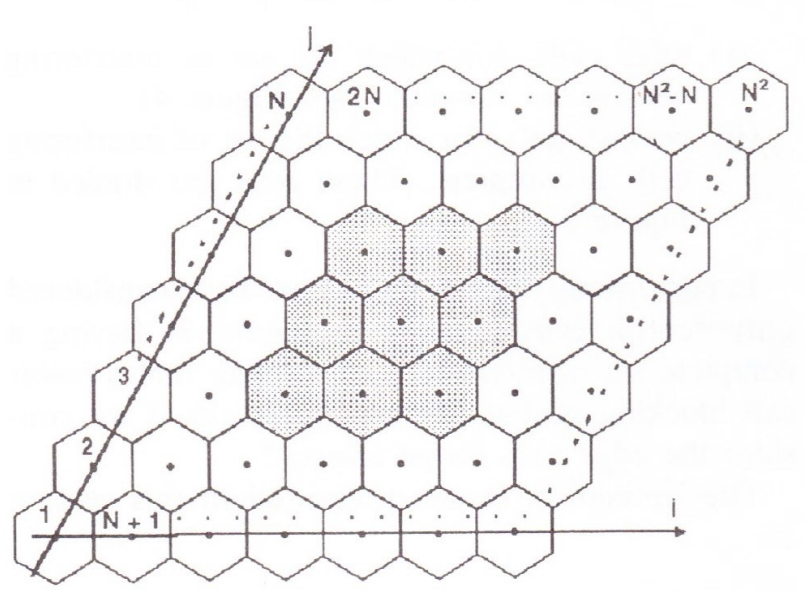

Figure 8. Simulated cellular network

have been obtained by assuming the following system parameters:

(a) $N=7$ (cells per side)

(b) set of interfering cells of $x$ formed by two tiers of adjacent cells of $x$ (i.e. a reuse of $7)^{3,5}$

(c) $M=70$ (channels).

Figure 9 shows the call blocking probability, $P_{\mathrm{b}}$, for the proposed DCA technique as a function of the offered traffic intensity per cell for the GEO case $(\alpha \rightarrow \infty)$.

In the same Figure, $P_{\mathrm{b}}$ achieved in the case of a FCA technique is also reported for comparison purposes. In this case a set of 10 channels is permanently assigned to each cell. The same set of channels is reused in cells at a distance $D$ away. The basic FCA technique implies that a call attempt at a cell site can only be served by an available channel belonging to the set of channels assigned to that cell site; otherwise the call is blocked and lost.

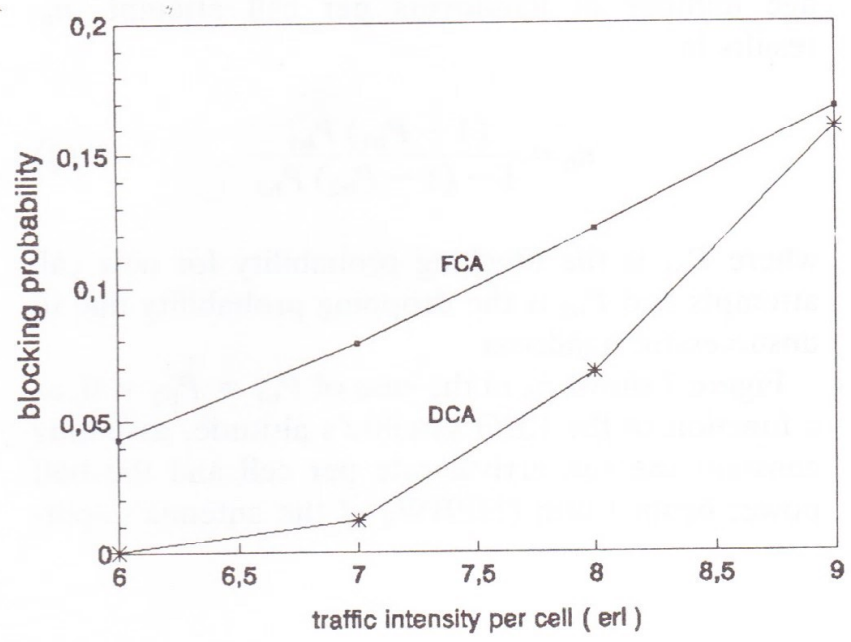

Figure 9. Blocking probability as a function of the offered traffic per cell, GEO case

According to the model outlined in the previous section the handover arrivals process may be modelled as a Poisson process, independent of the new call arrivals process and with average arrival rate $\lambda_{\mathrm{h}}$ related to $\lambda$ by

$$
\lambda\left(1-P_{\mathrm{b} 1}\right) P_{\mathrm{h} 1}+\lambda_{\mathrm{h}}\left(1-P_{\mathrm{b} 2}\right) P_{\mathrm{h} 2}=\lambda_{\mathrm{h}}
$$

Equation (10) has been derived by considering a stability condition which, in our case, means that the rate of mobile users leaving a cell is equal to the rate of mobile users entering the cell.

Note that from (9) and (10) we have

$$
\frac{\lambda_{\mathrm{h}}}{\lambda}=n_{\mathrm{h}}
$$

From above, it follows that $P_{\mathrm{b}}$ in the case of 


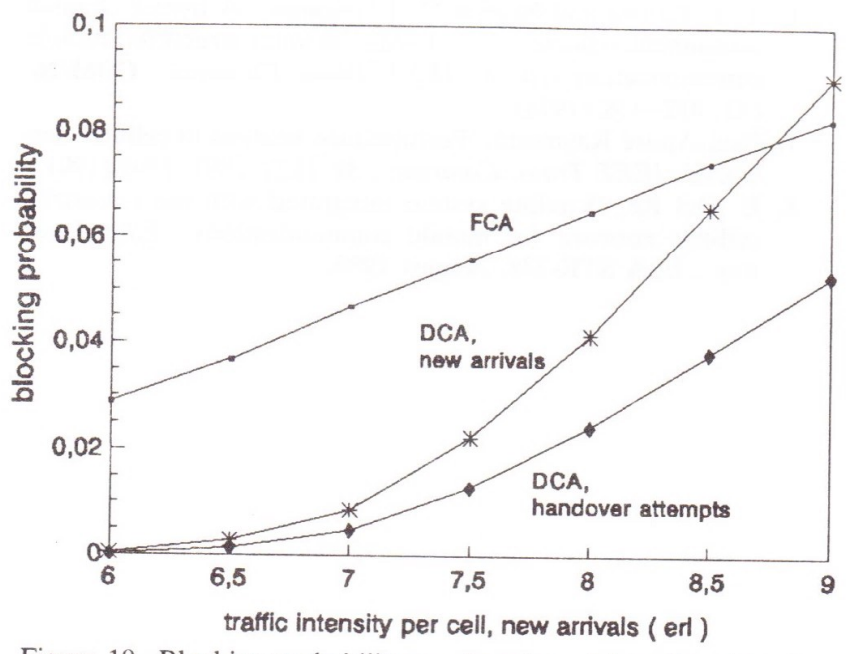

Figure 10. Blocking probability as a function of the offered traffic per cell, IRIDIUM case

the FCA technique can be theoretically derived by means of the ERLANG-B formula.

Figure 9 highlights the better performance of the DCA technique. These advantages are reduced under high tanic load conditions (network congestion $)^{7}$

Figures 10 and 11 show the behaviours of the blocking probability (both for new call attempts and for handover attempts) as a function of the offered traffic intensity per cell (new arrivals), for DCA and FCA in the case of IRIDIUM system (Figure 10 ) with $\alpha=0.32$ and the ARIES system (Figure 11) with $\alpha=0.47$. These two different values of $\alpha$ have been derived by assuming the same antenna HPBW

The considerations we can obtain from these Figures are

\section{GEO satellite system:}

The DCA technique outperforms the FCA technique, under practical call blocking probability values $(\leq 0 \cdot 03)$.

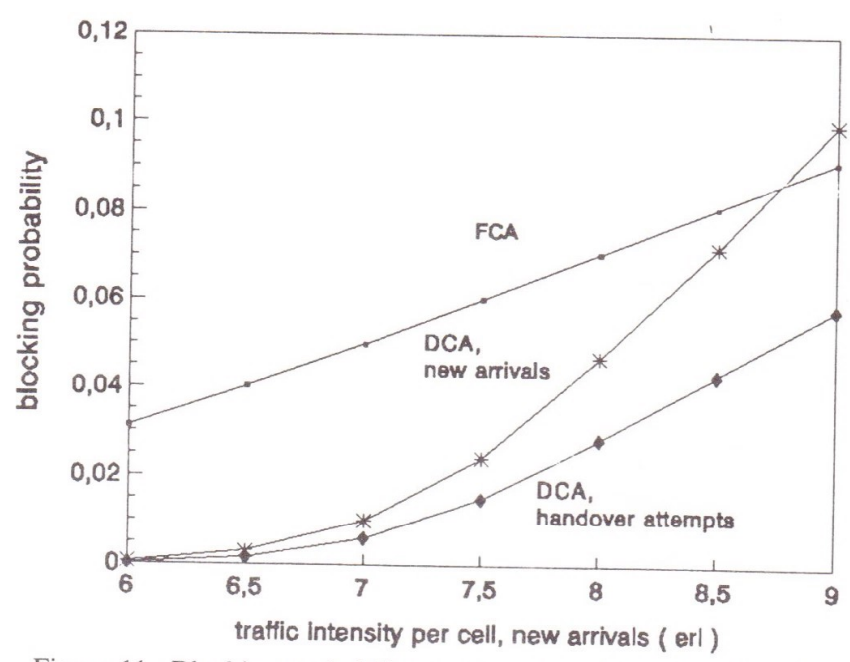

Figure 11. Blocking probability as a function of the offered traffic per cell, ARIES case

\section{LEO satellite systems}

1. If the users mobility increases (decreasing $\alpha$ ), the blocking probability decreases at the same traffic load, both in the case of FCA and DCA techniques.

2. The proposed DCA technique reduces the blocking probability of handover attempts with respect to that of new call attempts by an efficient use of the dynamic channel de-allocation algorithm outlined in Section 2. This is of particular importance in the case of LEO satellite systems due to the high value of the mean number of handovers per call (Figure 7). An analogous advantage cannot be obtained by the FCA technique, because the call management in a cell is independent from what happens in the adjacent cells.

\section{CONCLUSIONS}

In this paper an efficient DCA technique suitable for application in mobile satellite systems has been presented.

The proposed DCA technique employs a cost function defined for each available channel to achieve an optimum channel allocation on demand. In addition to this, a suitable channel de-allocation is performed whenever a call termination occurs in a cell to increase the spectrum efficiency.

It has been shown by computer simulations that the novel DCA technique achieves better performance than FCA for both the considered GEO and LEO satellite systems. In particular, in the case of LEO satellite systems it has been shown that the DCA technique permits a great reduction in the probability that a call in progress be dropped. This is of remarkable importance to achieve a good quality of service when the number of handovers per call is high, as in the LEO satellite systems. The results in Figures 9-11 also show that a target quality of service (e.g. a blocking probability not greater than 0.03 ) can be guaranteed for a much higher offered traffic intensity with DCA with respect to FCA. Assuming a traffic load of $20 \mathrm{mErl}$ per MS, 8 this results in a much greater mean number of MSs in service per cell under the specified target condition. In particular we have $300 \mathrm{MSs}$ for the FCA technique and 375 MSs for the DCA technique in the case of the IRIDIUM system (Figure 10).

\section{ACKNOWLEDGEMENT}

Work carried out under the financial support of the Italian Space Agency (ASI) and MURST.

\section{REFERENCES}

1. G. Maral, J. J. De Ridder, B. G. Evans and M. Richharia, 'Low earth orbit satellite systems for communications', Int. J. Satell. Commun., 9, 209-225 (1991). 
2. J. J. H. Lodge, 'Mobile satellite communication systems: toward global personal communications', IEEE Comm. Mag., 29, 24-30 (1991)

3. S. J. Campanella, 'Satellite switches', in E. Del Re, P. Bartholomè and P.P. Nuspl (eds), Satellite Integrated Communication Networks, North Holland, 1988, pp. 57-60.

4. V. H. MacDonald, 'The cellular concept', Bell Syst. Tech. J., 58, 15-41 (1979)

5. S. Tekinay and B. Jabbari, 'Handover and channel assignment in mobile cellular networks', IEEE Comm. Mag., 29 (11), 42-46 (1991)
6. T. J. Kahwa and Nicolas D. Georganas, 'A hybrid channel assignment scheme in large-scale, cellular-structured mobile communication system', IEEE Trans. Commun., COM-26. (4), 432-438 (1978)

7. Paul-André Raymond, 'Performance analysis of cellular networks', IEEE Trans. Commun., 39, (12), 1787-1793 (1991

8. E. Del Re, 'Satellite system integrated with the terrestrial cellular network for mobile communications', ESA Tech. Rep., ESA STR-228, August 1989. 